Comment. Math. Helv. 75 (2000) 201-215

(C) 2000 Birkhäuser Verlag, Basel

$0010-2571 / 00 / 020201-15 \$ 1.50+0.20 / 0$

Commentarii Mathematici Helvetici

\title{
A geometric characteristic splitting in all dimensions
}

\author{
Bernhard Leeb* and Peter Scott ${ }^{\dagger}$
}

\begin{abstract}
We prove the existence of a geometric characteristic submanifold for non-positively curved manifolds of any dimension greater than or equal to three. In dimension three, our result is a geometric version of the topological characteristic submanifold theorem due to Jaco, Shalen and Johannson.
\end{abstract}

Mathematics Subject Classification (2000). 53C20, 57M50.

Keywords. Nonpositive curvature, JSJ decomposition, characteristic submanifold.

\section{Introduction}

In the 1970's, Jaco and Shalen [JS] and Johannson [J] showed that a closed orientable Haken 3-manifold $M$ has a canonical family of disjoint embedded incompressible tori, no two of which are parallel, such that the complementary pieces of $M$ are either Seifert fibre spaces or are atoroidal. They defined the characteristic submanifold $V(M)$ of $M$ to be essentially the union of the Seifert manifold pieces of $M$. Further, they showed that any essential map of the torus into $M$ is homotopic into $V(M)$. Johannson called this last property the Enclosing Property. For brevity, we will refer to these results as the JSJ results.

In this paper, we show that if $M$ is a closed manifold of dimension three or more, and if $M$ has a Riemannian metric of non-positive curvature, then either the metric on $M$ is flat or there is a precisely analogous decomposition of $M$ along codimension one submanifolds. Further these submanifolds are totally geodesic in $M$ and are flat in the metric induced from $M$. Note that in dimension three, a flat manifold must be a Seifert fibre space, so that, in particular, our arguments give a new proof of the JSJ results for the special case when $M$ is assumed to have a metric of non-positive curvature. In dimension four or more, a flat manifold need not be a Seifert manifold, see the example near the end of section 1, so this case really is different in higher dimensions. We also prove that essentially the same

${ }^{*}$ Partially supported by SFB 256 (Bonn) and IHES

${ }^{\dagger}$ Partially supported by NSF grant DMS 9306240 
results hold if $M$ is non-orientable and if the boundary of $M$ is non-empty, on the assumption that the boundary is convex.

At the time when Jaco and Shalen and Johannson proved their results, the methods seemed very special to dimension three and no one even asked whether this result had any generalization to higher dimensions. Several years later in 1990, Kropholler [Kr] published an algebraic analogue of their results. He considered Poincaré duality groups of dimension three ( $P D 3$-groups). The fundamental group of any closed aspherical 3-manifold is automatically a $P D 3$-group, but it is not known whether the converse holds. Note, however, that $P D 2$-groups are known to be fundamental groups of closed surfaces, so it is not unreasonable to hope that every $P D 3$-group is the fundamental group of an aspherical closed 3 -manifold. There is a natural analogue, in the context of $P D 3$-groups, of an embedded incompressible surface. One considers a $P D 3$-group $G$ and a subgroup $H$ such that $H$ is a $P D 2$-group and $G$ splits over $H$, i.e. $G$ can be expressed as $A *_{H}$, or as $A *_{H} B$ with $A \neq H \neq B$. Kropholler showed that the natural analogue of the JSJ splitting result holds for $P D 3$-groups. Surprisingly, he also showed that his algebraic result had a generalization to $P D n$-groups in all dimensions greater than three. This raised the question of whether the topological results of Jaco, Shalen and Johannson also generalized to higher dimensions. As Haken manifolds are aspherical and Kropholler's results correspond to results about aspherical manifolds, it seems possible that the JSJ results might generalise to aspherical manifolds but not to all manifolds. Note that a Riemannian manifold of non-positive curvature with convex boundary is aspherical, so our results show that the JSJ results generalize to aspherical manifolds in the special case of non-positive curvature.

Jaco and Shalen [JS] and Johannson [J] also considered non-closed manifolds and defined a characteristic submanifold $V(M)$ for any orientable Haken 3-manifold $M$ with incompressible boundary. They showed that such a manifold $M$ has a canonical family of disjoint properly embedded incompressible tori and annuli, no two of which are parallel. They defined the characteristic submanifold $V(M)$ of $M$ to be essentially the union of the Seifert manifold pieces of $M$ together with some pieces which are homeomorphic to $I$-bundles. Further, they showed that any essential map of the torus or annulus into $M$ is homotopic into $V(M)$. In this paper, we give analogous results for compact manifolds with non-empty boundary in any dimension greater than or equal to three, but we assume that $M$ has a metric of non-positive curvature and that the boundary of $M$ is totally geodesic.

The results in this paper were proved by the authors independently in 1992. At about the same time, Sela $[\mathrm{S}]$ announced some algebraic results which are closely related to all the preceding discussion. Sela's results were for negatively curved groups and were the precise analogue of the JSJ results for the case of 3-manifolds with no incompressible tori. This is because a negatively curved group cannot have a subgroup isomorphic to $\mathbf{Z} \times Z$. The topological picture is of a 3-manifold with a canonical family of disjoint embedded annuli, and Sela's picture is of a group which splits over several different infinite cyclic subgroups. Sela's Enclosing 
Property is the analogue of the JSJ Enclosing Property for embedded annuli only. More recently, Rips and Sela [RS] have announced a generalization of Sela's results to cover all finitely presented groups. Thus again we are left with the question of whether the results in this paper can be generalised to manifolds which need not have non-positive curvature.

As we pointed out earlier, a Riemannian manifold $M$ of non-positive sectional curvature with convex boundary is aspherical and so its homotopy type is determined by its fundamental group. It is well-known that various algebraic properties of $\pi_{1}(M)$ have strong implications for the geometry of $M$. The most basic example, due to Gromoll-Wolf [GW] and Lawson-Yau [LY], is that an abelian subgroup of $\pi_{1}(M)$ is carried by a totally-geodesically immersed flat torus. In this paper, we obtain information about the geometric structure of non-positively curved manifolds from the intersection pattern of the closed flat totally-geodesic hypersurfaces. Our main results are geometric versions of the topological decomposition theorem in dimension three due to Jaco, Shalen [JS] and Johannson [J].

Geometric Decomposition Theorem in Dimension Three. Let $M$ be a compact connected non-positively curved 3-manifold which has convex boundary. Then either $M$ is closed and has a flat metric, or $M$ can be canonically decomposed along finitely many totally-geodesically embedded flat 2-tori and Klein bottles. The resulting pieces are Seifert or atoroidal. Further any $\pi_{1}$-injective map of the torus or Klein bottle into $M$ can be homotoped to a totally geodesic flat immersion, and any such immersion must lie in one of the Seifert pieces or be homotopic to a cover of one of the decomposing surfaces.

Note that some of the decomposing surfaces may be one-sided. In particular, no piece in the decomposition of $M$ will be an interval bundle over a flat surface unless $M$ itself is an interval bundle over a flat surface. If $M$ is a twisted interval bundle over a flat surface $F$ then our construction splits $M$ along the one-sided surface $F$.

The Seifert pieces of $M$ admit a Seifert fibration by closed geodesics and they are rigid in the sense that they split locally as a Riemannian product, the fiber being the one-dimensional factor. Note that if $M$ is flat, it is also Seifert fibered in the three dimensional case. The proof of our theorem readily applies to all dimensions. See the end of section 1 for the definitions.

Geometric Decomposition Theorem. Let $M$ be a compact connected nonpositively curved manifold of dimension $n \geq 3$, which has convex boundary. Then either $M$ is closed and has a flat metric, or $M$ can be canonically decomposed along finitely many totally-geodesically embedded flat closed submanifolds of codimension one. The resulting pieces are Seifert fibered or codimension-one atoroidal. Further any essential map of a closed flat $(n-1)$-manifold into $M$ can be homotoped to a totally geodesic flat immersion, and any such immersion must lie in one of the 
Seifert pieces or be homotopic to a cover of one of the decomposing hypersurfaces.

As in the three-dimensional case, some of the decomposing hypersurfaces may be one-sided. There is also a more general version of this result which corresponds to the full JSJ splitting of an orientable compact 3-manifold along annuli as well as tori. We leave the statement to section 4.

This paper is organized as follows: In section 2 we prove that there is an upper bound for the number of mutually non-parallel, disjoint, totally-geodesically embedded, closed hypersurfaces in a compact non-positively curved manifold $M$ with convex boundary. In section 3 , we study the pattern $\mathcal{S}$ of totally-geodesically immersed, flat, closed hypersurfaces in $M$. We show that intersecting hypersurfaces span a geometric Seifert submanifold. The desired decomposition of $M$ is obtained by cutting along hypersurfaces in $\mathcal{S}$ which are isolated in the sense that they do not intersect any other surface in $\mathcal{S}$. In section 4 , we discuss how to prove the most general version of our results.

\section{Preliminaries}

Non-positive curvature. We start by recalling a few well-known facts from the geometry of nonpositively curved manifolds, for more details the reader may consult e.g. [ChE]. In this paper, we consider smooth Riemannian manifolds of non-positive sectional curvature. We will always assume that they are complete as metric spaces and that their boundaries are convex, i.e. each geodesic touching the boundary must already be contained in the boundary. A simply-connected manifold $X$ of this kind has the fundamental property that its distance function $d: X \times X \rightarrow \mathbb{R}$ is convex, that is, for any two geodesics $c_{1}, c_{2}:[a, b] \rightarrow X$ the function $t \rightarrow d\left(c_{1}(t), c_{2}(t)\right)$ is convex. In particular, the distance $d(\cdot, C)$ from a convex subset $C \subset X$ is a convex function. The convexity of $d$ implies that any two points in $X$ can be connected by a unique geodesic. This has strong topological implications: $X$ is contractible so that any manifold $M$ covered by $X$ is aspherical, i.e. $M$ is a $K(\pi, 1)$-space.

A smooth submanifold $Y \subseteq X$, possibly with boundary, is called totallygeodesic if each geodesic in $X$ tangent to an interior point of $Y$ belongs locally to $Y$. We call $Y$ (geodesically) complete if each geodesic in $Y$ is extendable ad infinitum. If $Y_{1}$ and $Y_{2}$ are complete totally-geodesic submanifolds of $X$ which have bounded distance from each other, then the distance functions $\left.d\left(\cdot, Y_{i}\right)\right|_{Y_{j}}$ are constant by convexity and completeness. This implies that the submanifolds $Y_{i}$ are parallel, i.e. there is a totally-geodesic submanifold in $X$ which splits metrically as $Y \times\left[a_{1}, a_{2}\right]$ so that $Y_{i}=Y \times\left\{a_{i}\right\}$.

For an isometry $\phi$ of $X$, denote by $\operatorname{MIN}(\phi)$ the set where the displacement function $d_{\phi}: x \rightarrow d(x, \phi x)$ assumes its infimum. Since $d$ is convex, $\operatorname{MIN}(\phi)$ is a closed convex subset of $X$. An isometry $\phi$ is called non-parabolic or semisimple 
if $\operatorname{MIN}(\phi)$ is non-empty. In this case, $\phi$ is elliptic if the minimum of $d_{\phi}$ equals zero and loxodromic if it is strictly positive. The set of minimal displacement for a loxodromic isometry splits metrically as

$$
\operatorname{MIN}(\phi) \cong \mathbb{R} \times Y
$$

where the lines $\mathbb{R} \times\{y\}$ are the $\phi$-axes, i.e. geodesics preserved by $\phi$, and $Y$ is a simply-connected manifold of nonpositive curvature with convex boundary. Isometries of $X$ commuting with $\phi$ preserve the splitting (1). It follows by induction that any abelian subgroup $A$ of the isometry group of $X$ preserves a flat in $X$ where a flat is defined to be a convex subset isometric to a Euclidean space. More precisely, the intersection of minimal sets $\bigcap_{\gamma \in A} \operatorname{MIN}(\gamma)=: \operatorname{MIN}(A)$ is non-empty and splits metrically as

$$
\operatorname{MIN}(A) \cong E \times Y
$$

where $E$ is a Euclidean space (possibly of dimension zero) and $Y$ is a simplyconnected manifold of nonpositive curvature with convex boundary. The layers $E \times\{y\}$ are the minimal $A$-invariant flats and the induced action of $A$ on $E$ is cocompact.

Suppose that $\Gamma$ is a group which acts properly-discontinuously and cocompactly by isometries on $X$, such as the group of deck-transformations corresponding to a compact Riemannian manifold covered by $X$. Let $A \subset \Gamma$ be an abelian subgroup (which is necessarily finitely generated as $X$ is non-positively curved) and denote by $C(A)$ its centraliser and by $N(A)$ its normaliser. The action of $N(A)$ on $X$ preserves $\operatorname{MIN}(A)$ and the splitting $(2)$.

Lemma 1.1. The action of $C(A)$ on $\operatorname{MIN}(A)$ is cocompact.

Proof. Let $\left(p_{n}\right)$ be a sequence of points in $\operatorname{MIN}(A)$. Since $\Gamma$ acts cocompactly on $X$, there exist isometries $\gamma_{n} \in \Gamma$ so that the sequence $\left(\gamma_{n} p_{n}\right)$ is bounded. Let $a_{1}, \ldots, a_{r}$ denote a basis of $A$. For each value of the index $i$, the points

$$
\gamma_{n} a_{i} \gamma_{n}^{-1} \cdot \gamma_{n} p_{n}=\gamma_{n} \cdot a_{i} p_{n}
$$

form a bounded sequence too, because $d\left(a_{i} p_{n}, p_{n}\right)$ equals the minimal displacement of the isometry $a_{i}$. Since the action of $\Gamma$ is properly discontinuous, the elements $\gamma_{n} a_{i} \gamma_{n}^{-1}$ are contained in a finite subset of $\Gamma$. By passing to a subsequence $r$ times, we can assume that, for each $i, \gamma_{n} a_{i} \gamma_{n}^{-1}$ is a fixed element of $\Gamma$ for all values of $n$. Let $\gamma_{n}^{\prime}$ denote $\gamma_{1}^{-1} \gamma_{n}$ which must lie in $C(A)$. Then the sequence $\left(\gamma_{n}^{\prime} p_{n}\right)$ is bounded, as it is obtained from the bounded sequence $\left(\gamma_{n} p_{n}\right)$ by applying $\gamma_{1}^{-1}$. Since $\left(p_{n}\right) \subset \operatorname{MIN}(A)$ was chosen arbitrarily, we conclude that there is a bounded fundamental domain for the action of $C(A)$ on $\operatorname{MIN}(A)$.

The following auxiliary result will be needed later: 
Lemma 1.2. Let $X_{1}$ and $X_{2}$ be simply-connected Riemannian manifolds of nonpositive curvature (metrically complete and with convex boundary). If $F \subset X_{1} \times X_{2}$ is a totally-geodesically embedded flat submanifold, then the images of $F$ under the projections $p_{i}: X_{1} \times X_{2} \rightarrow X_{i}$ on the factors are also flat.

Proof. Let $c$ and $c^{\prime}$ be geodesic segments in $F$ so that their distance function $d(t):=d\left(c(t), c^{\prime}(t)\right)$ is constant. Denote by $d_{i}$ the distance function of the projected segments $p_{i} \circ c$ and $p_{i} \circ c^{\prime}$. Then $d^{2}=d_{1}^{2}+d_{2}^{2}$. Since the $d_{i}$ are convex, $d^{2}$ can only be constant if the $d_{i}$ are constant. Hence $p_{i}$ maps parallel segments to parallel segments and the claim follows.

Topology. We explain the notions necessary to state the topological decomposition theorem due to Jaco, Shalen and Johannson. We work in the smooth category. Let $M$ be a compact orientable 3-manifold, possibly with boundary, which is irreducible, i.e. every embedded 2-sphere bounds an embedded 3-ball, and has infinite fundamental group. We consider connected, two-sided, embedded surfaces $\Sigma$ in $M$ which are not homeomorphic to the 2-sphere. We will also require that $\Sigma$ be properly embedded in $M$ or be embedded in the boundary of $M$. Such a surface $\Sigma$ is called incompressible if there is no disc $D$ embedded in $M$ such that $D \cap \Sigma=\partial D$ and $\partial D$ is a non-contractible curve in $\Sigma$. If $M$ contains a properly embedded incompressible surface, then $M$ is a Haken manifold. The following decomposition theorem has been proven for Haken manifolds by Jaco, Shalen [JS] and Johannson $[\mathrm{J}]$. The non-Haken case follows from the fact that if a compact orientable irreducible 3 -manifold admits a $\pi_{1}$-injective map of the torus but does not admit such an embedding, then it must be a Seifert fibre space. This result requires the work of several authors and the proof was completed independently by Casson and Jungreis [CJ] and Gabai [Ga].

Topological Decomposition Theorem. A compact orientable irreducible 3manifold with infinite fundamental group and incompressible boundary can be cut along finitely many disjoint incompressible 2-tori into atoroidal and Seifert pieces, and any $\pi_{1}$-injective map of the 2-torus into the manifold is homotopic into one of the Seifert pieces or to a covering of one of the decomposing tori. Moreover, a minimal such decomposition is unique up to isotopy.

It remains to explain the types of pieces which occur: These are compact 3-manifolds $N$ with boundary. A 3-manifold $N$ is atoroidal if any $\pi_{1}$-injective map of the torus into $N$ is homotopic into the boundary of $N$. It is a Seifert manifold if it admits a Seifert fibration, i.e. if it can be expressed as a disjoint union of embedded circles, the fibres, so that the following is true: Every fibre has a neighborhood which is isomorphic, as a fibred space, to a fibred solid torus or Klein bottle. A fibred solid torus is a quotient of the trivially fibred product $D^{2} \times \mathbb{R}$ by a diffeomorphism $(\phi, \tau)$ where $\phi$ is an isometry of finite order of the 
unit disc $D^{2}$ and $\tau$ is a translation on the real line.

We also need to define what is meant by the terms Seifert manifold and atoroidal in higher dimensions. In dimension three, a Seifert manifold is a Seifert bundle over a 2-dimensional orbifold with fiber the circle. In the context of this paper, we define a Seifert manifold $N$ of dimension $n$ to be a Seifert bundle over a 2-dimensional orbifold with fiber a flat $(n-2)$-manifold. This means that $N$ is foliated by $(n-2)$-dimensional closed flat manifolds so that each leaf has a foliated neighborhood which has a finite cover whose induced foliation is a product $F \times D^{2}$. A manifold $M$ of dimension $n$ is codimension-one atoroidal if any $\pi_{1}$-injective map of a flat $(n-1)$-torus into $M$ is homotopic into the boundary of $M$.

\section{Immersed totally-geodesic submanifolds}

From now on, $M$ will denote a compact connected Riemannian manifold $M$ of nonpositive curvature with convex boundary. We denote by $\pi: \tilde{M} \rightarrow M$ the universal covering map and think of $\pi_{1}(M)=: \Gamma$ as the group of deck transformations acting on $\tilde{M}$.

Let $\phi: \Sigma \rightarrow M$ be a totally-geodesic Riemannian immersion of a closed connected non-positively curved manifold $\Sigma$ into $M$. Every lifting to a map of universal covers is a totally-geodesic embedding $\tilde{\phi}: \tilde{\Sigma} \hookrightarrow \tilde{M}$ and induces an injective homomorphism $\pi_{1}(\Sigma) \hookrightarrow \Gamma=\pi_{1}(M)$ of fundamental groups. Different lifts yield conjugate subgroups of $\Gamma$. Note that $\tilde{\Sigma}$ is geodesically complete.

\subsection{Intersections}

Lemma 2.1. Let $C_{1}, C_{2} \subset \tilde{M}$ be closed subsets so that the stabiliser $\Gamma_{i}:=$ $\operatorname{Stab}_{\Gamma}\left(C_{i}\right)$ acts cocompactly on $C_{i}$. Then $\Gamma_{1} \cap \Gamma_{2}$ acts cocompactly on $C_{1} \cap C_{2}$.

Proof. The natural map $\left(\Gamma_{1} \cap \Gamma_{2}\right) \backslash \Gamma_{2} \rightarrow \Gamma_{1} \backslash \Gamma$ is injective and the corresponding immersion $\left(\Gamma_{1} \cap \Gamma_{2}\right) \backslash C_{2} \rightarrow \Gamma_{1} \backslash \tilde{M}$ is therefore proper. Hence the inverse image under this immersion of the compact subset $\Gamma_{1} \backslash C_{1}$ is compact. As this inverse image equals $\left(\Gamma_{1} \cap \Gamma_{2}\right) \backslash\left(C_{1} \cap C_{2}\right)$, the lemma follows.

Note that the lemma holds more generally for properly discontinuous group actions on locally-compact topological spaces.

Corollary 2.2. Let $\Sigma_{1}$ and $\Sigma_{2}$ be closed non-positively curved Riemannian manifolds and suppose that $\phi_{1}: \Sigma_{1} \rightarrow M$ and $\phi_{2}: \Sigma_{2} \rightarrow M$ are totally-geodesic Riemannian immersions. Then $\phi_{1}\left(\Sigma_{1}\right) \cap \phi_{2}\left(\Sigma_{2}\right)$ is a finite union of totally-geodesically immersed closed non-positively curved Riemannian manifolds.

Proof. The immersion $\phi_{i}$ lifts to an embedding of universal covers with image a closed convex subset $Y_{i} \subset \tilde{M}$. By the previous lemma, the totally-geodesic 
submanifolds $\gamma_{1} \cdot Y_{1} \cap \gamma_{2} \cdot Y_{2}, \gamma_{1}, \gamma_{2} \in \Gamma$, have cocompact stabilisers in $\Gamma$. The corollary follows because, by compactness, $\phi_{1}\left(\Sigma_{1}\right) \cap \phi_{2}\left(\Sigma_{2}\right)$ is the projection of finitely many submanifolds $\gamma_{1} \cdot Y_{1} \cap \gamma_{2} \cdot Y_{2}$.

\subsection{Finiteness for disjoint non-parallel totally-geodesic hypersurfaces}

Definition 2.3. We call two totally-geodesic Riemannian immersions $\phi_{1}: \Sigma_{1} \rightarrow$ $M$ and $\phi_{2}: \Sigma_{2} \rightarrow M$ of closed non-positively curved manifolds into $M$ parallel if there are a totally-geodesic embedding $\Phi: \tilde{\Sigma}_{1} \times\left[a_{1}, a_{2}\right] \hookrightarrow \tilde{M}$ and Riemannian covering maps $p_{i}: \tilde{\Sigma}_{1} \times\left\{a_{i}\right\} \rightarrow \Sigma_{i}$ such that $\phi_{i} \circ p_{i}=\left.\pi \circ \Phi\right|_{\tilde{\Sigma}_{1} \times\left\{a_{i}\right\}}$.

If we have two totally-geodesic Riemannian immersions of $\Sigma$ into $M$ which are homotopic, then there will be totally-geodesic submanifolds $Y$ and $Y^{\prime}$ in $\tilde{M}$ covering these immersions and lying a bounded distance apart. Thus $Y$ and $Y^{\prime}$ are parallel, and hence so are the two immersions of $\Sigma$ into $M$.

Our aim is to prove the following result.

Proposition 2.4. Let $M$ be a compact non-positively curved Riemannian manifold with convex boundary. Then there is an upper bound to the number of disjoint, closed, totally-geodesically embedded hypersurfaces in $M$ so that no two of them are parallel.

As discussed above, any such hypersurface is $\pi_{1}$-injective and if two such hypersurfaces are homotopic, they must be parallel. Now in the topological setting in dimension three, it is a standard result $[\mathrm{H}]$ that, in any compact 3-manifold $M$, there is an upper bound to the number of disjoint, embedded, $\pi_{1}$-injective closed surfaces in $M$ which are pairwise non-parallel, where two surfaces $S$ and $S^{\prime}$ are parallel if they together bound a submanifold homeomorphic to $S \times I$. This upper bound is called the Haken number of $M$. In higher dimensions, there is no such result in the general topological setting, but there is an algebraic analogue due to Dunwoody [D], which discusses splittings of $P D n$-groups over $P D(n-1)$ subgroups. This implies that if one considers a closed aspherical manifold $M$, there is an upper bound to the number of disjoint closed aspherical $\pi_{1}$-injective embedded codimension-one submanifolds in $M$ such that no two are homotopic. Clearly this result will also apply to any aspherical compact manifold with boundary so long as the boundary is also $\pi_{1}$-injective and aspherical. Now the hypotheses of the above proposition imply that $M$ is aspherical and that its boundary is $\pi_{1}$-injective and aspherical. Thus one can prove this proposition in dimension three by using the Haken number, and can prove it in any dimension by using Dunwoody's result. However, we will give a direct geometric proof.

Proof of Proposition 2.4. Let $\Sigma_{1}, \ldots, \Sigma_{n}$ be such a family of hypersurfaces. It is possible that some of these hypersurfaces are components of $\partial M$. Consider a 
component $N$ of $M \backslash \cup_{i=1}^{n} \Sigma_{i}$. Identify the universal cover $\tilde{N}$ with a component of $\pi^{-1}(N) \subset \tilde{M}$. We will use $\partial \tilde{N}$ to denote the boundary of $\overline{\tilde{N}}$ as a manifold. Then $\partial \tilde{N}$ consists of a union of components of $\partial \tilde{M}$ and of totally geodesic hypersurfaces in $\tilde{M}$ each of which covers one of the surfaces $\Sigma_{i}$. Also $\partial \tilde{N}$ consists of at least two components. For otherwise, $N$ would have infinite diameter contradicting the compactness of $M$.

Consider the case that $\partial \tilde{N}$ has exactly two components $Y_{1}$ and $Y_{2}$. At least one of them, say $Y_{1}$, covers a hypersurface which we denote $\Sigma_{1}$. The distance function $d\left(\cdot, Y_{1}\right)$ is bounded on $Y_{2}$, and vice versa, because the subgroup of $\Gamma$ preserving $\tilde{N}$ and $\frac{Y_{1}}{\hat{N}}$ also preserves $Y_{2}$ and acts cocompactly on $\overline{\tilde{N}}$. Thus $Y_{1}$ and $Y_{2}$ are parallel and $\bar{N}$ is isometric to a product $Y_{1} \times[-a, a]$. If also $Y_{2}$ covers a hypersurface $\Sigma_{i}$, then $\Sigma_{i}$ is parallel to $\Sigma_{1}$ and hence $\Sigma_{i}=\Sigma_{1}$ by our assumption. If $\overline{\tilde{N}}$ projects onto $M$, then $n=1$. Otherwise the image of $\overline{\tilde{N}}$ is a twisted interval bundle over the hypersurface $\Sigma_{1}^{\prime}$ covered by $Y_{1} \times\{0\}$. In this case, we replace $\Sigma_{1}$ by $\Sigma_{1}^{\prime}$. If $Y_{2}$ covers no hypersurface $\Sigma_{i}$ and hence $Y_{2} \subset \partial \tilde{M}$, then we remove $N$ from $M$. In both cases, this reduces by one the number of components of $M \backslash \cup_{i=1}^{n} \Sigma_{i}$ and does not alter the number of hypersurfaces $\Sigma_{i}$. By repeating these steps, we may assume that for all pieces $N$, the universal cover $\tilde{N}$ has at least three boundary components. The pieces then have a certain minimal size:

Lemma 2.5. Each component $N$ of $M \backslash \cup_{i=1}^{n} \Sigma_{i}$ contains a point $p$ at distance at least $\rho_{0}$ from the boundary $\partial N$, where $\rho_{0}$ is a positive constant only depending on the lower sectional curvature bound of $M$.

Proof. We re-scale so that the sectional curvature of $M$ is bounded by $-1 \leq K_{M} \leq$ 0 . Let $\tilde{p} \in \tilde{N}$ be a point at maximal distance $\rho$ from $\partial \tilde{N}$. The ball $B$ of radius $\rho$ centered at $\tilde{p}$ touches three components $Y_{1}, Y_{2}, Y_{3}$ of $\partial \tilde{N}$ in respective points $\tilde{p}_{1}, \tilde{p}_{2}, \tilde{p}_{3}$. Let $v_{i}$ be the unit vector in $\tilde{p}$ pointing in the direction of $\tilde{p}_{i}$. Among the vectors $v_{1}, v_{2}, v_{3}$ at least two, say $v_{1}$ and $v_{2}$, enclose an angle $\angle\left(v_{1}, v_{2}\right) \leq \frac{2}{3} \pi$. Consider the arc in the unit sphere in $T_{\tilde{p}} \tilde{M}$ joining $v_{1}$ and $v_{2}$. It contains a vector $v$ such that the geodesic ray $r:[0, \infty) \rightarrow \tilde{M}$ emanating from $\tilde{p}$ in the direction of $v$ intersects neither $Y_{1}$ nor $Y_{2}$. We assume without loss of generality that $\angle\left(v, v_{1}\right) \leq \frac{1}{3} \pi$. The angles of the triangles $\tilde{p} \tilde{p}_{1} r(t)$ satisfy for all $t>0$ :

$$
\angle_{\tilde{p}}\left(\tilde{p}_{1}, r(t)\right) \leq \frac{1}{3} \pi, \quad \angle_{\tilde{p}_{1}}(\tilde{p}, r(t)) \leq \frac{1}{2} \pi
$$

Consider comparison triangles with the same side lengths in the hyperbolic plane $\mathbb{H}^{2}$. By Toponogov's triangle comparison theorem $[\mathrm{K}]$, the angles in the comparison triangles are not greater than the corresponding angles in the triangles $\tilde{p} \tilde{p}_{1} r(t)$. So they satisfy analogous inequalities. Since $t$ may be arbitrarily large, we can bound $\rho$ from below by a positive constant $\rho_{0}$, namely by the finite sidelength of the triangle in $\mathbb{H}^{2}$ with angles $0, \frac{1}{3} \pi, \frac{1}{2} \pi$ and one ideal vertex. 
Denote by $c(n)$ the number of components of $M \backslash \cup_{i=1}^{n} \Sigma_{i}$. According to Lemma 2.5, there is a $2 \rho_{0}$-net in $M$ with one point in each component of $M \backslash \cup_{i=1}^{n} \Sigma_{i}$. By compactness of $M, c(n)$ stabilizes as $n$ tends to infinity. More precisely, it can be bounded above in terms of the lower curvature bound and the volume of $M$. If $c\left(n_{1}\right)=c\left(n_{2}\right)$ for $n_{1}<n_{2}$, then we can choose for each $n$ with $n_{1}<n \leq n_{2}$ a closed smooth path $\alpha_{n}$ which does not intersect $\Sigma_{1}, \ldots, \Sigma_{n-1}$ but intersects $\Sigma_{n}$ once transversally. Looking at the intersection numbers modulo 2 of the paths $\alpha_{n}$ with the surfaces $\Sigma_{n}$, we see that the $\alpha_{n}$ represent linearly independent homology classes in $H_{1}(M, \mathbb{Z} / 2 \mathbb{Z})$. Since $M$ is compact, we conclude that $n_{2}-n_{1}$ is bounded in terms of the topology of $M$. This completes the proof of Proposition 2.4.

\section{Geometric decomposition along closed submanifolds}

In this section, $M$ will always denote a compact, connected, non-positively curved Riemannian manifold of dimension at least 3 which has convex boundary. We investigate how the pattern of closed totally-geodesic flat hypersurfaces in $M$ is organized to yield a canonical geometric decomposition. In dimension three this is a geometric realization of the canonical topological decomposition due to Jaco, Shalen and Johannson. The decomposition of $M$ will be obtained by cutting along hypersurfaces of the following kind (see section 3.2):

Definition 3.1. A totally-geodesically immersed, closed, flat hypersurface in $M$ is called isolated if it does not intersect any such hypersurface transversally.

Note that the definition also excludes self-intersections. It is immediate that isolated closed flat hypersurfaces cover embedded hypersurfaces and the images of two of them must coincide or be disjoint.

Denote by $\Gamma$ the fundamental group of $M$ thought of as a group of deck transformations acting on $\tilde{M}$. Closed flat hypersurfaces in $M$ are covered by $(n-1)$-flats in $\tilde{M}$ which are periodic in the sense of:

Definition 3.2. A $\Gamma$-periodic flat or $\Gamma$-flat is a flat $F$ in $\tilde{M}$ such that the subgroup $\Gamma_{F}$ of $\Gamma$ preserving $F$ acts cocompactly on $F$. We call $F$ isolated if it intersects no other $\Gamma$-flat transversally.

Unless explicitly stated otherwise, all flats considerd in this section will be $(n-1)$-dimensional. A totally-geodesically immersed closed flat hypersurface is isolated if and only if it is covered by isolated $\Gamma$-flats in $\tilde{M}$.

\subsection{Seifert fibred submanifolds}

From now on we will assume that $M$ is not closed and flat. We prove in this section that intersecting, totally-geodesically immersed, closed, flat hypersurfaces 
in $M$ span a submanifold which is foliated by parallel closed flat submanifolds of codimension two. In dimension three, this foliation is a Seifert fibration by closed geodesics. Our arguments are closely related to those in Casson's proof of the Torus Theorem in dimension three $[\mathrm{C}]$, but are simpler because of the curvature assumption which we are imposing on the metric of $M$.

Let $A \subset \Gamma$ be a free abelian subgroup of rank $n-2$. Recall from section 1 that the normaliser $N(A)$ of $A$ in $\Gamma$ acts cocompactly on the set of minimal displacement $\operatorname{MIN}(A)$ and preserves its metric splitting (2). The induced action of $A$ on $E$ is cocompact, so $E$ is Euclidean space of dimension $n-2$.

Now let $H_{A}$ denote the closed convex hull of the union of all $A$-invariant $\Gamma$ flats. $H_{A}$ is $A$-invariant and hence has the form $H_{A}=Z \times \mathbb{R} \subseteq Y \times \mathbb{R}=\operatorname{MIN}(A)$ for a closed convex subset $Z$ of $Y$. Furthermore $H_{A}$ is preserved by $N(A)$ and Lemma 1.1 implies that the action of $N(A)$ on $H_{A}$ is cocompact. Note that it is possible that $H_{A}$ consists of a single $A$-invariant $\Gamma$-flat and so has empty interior. In this case it will be convenient to write $\partial H_{A}=H_{A}$.

Lemma 3.3. The boundary $\partial H_{A}$ is a disjoint union of $\Gamma$-flats.

Proof. Each $A$-invariant $\Gamma$-flat projects to a complete geodesic in $Y$. Let $Z$ denote the closed convex hull of the family $\mathcal{F}$ of all such geodesics, so that $Z$ is either a geodesic or a convex subset of $Y$ with non-empty interior whose boundary $\partial Z$ is a union of disjoint complete geodesics. Consequently, $\partial H_{A}$ is a disjoint union of $A$-invariant $(n-1)$-flats lying above $\partial Z$. According to Lemma 1.1 the quotient manifold $N(A) \backslash H_{A}$ is compact and therefore also its closed subset $\partial\left(N(A) \backslash H_{A}\right)=$ $N(A) \backslash \partial H_{A}$. Hence the components of $\partial H_{A}$ are $\Gamma$-flats.

Next we consider how codimension-one flats in $\tilde{M}$ can meet $\operatorname{MIN}(A)$.

Lemma 3.4. Suppose that $\operatorname{MIN}(A)$ contains two non-parallel $A$-invariant $\Gamma$-flats. Then any codimension-one flat intersecting $\operatorname{MIN}(A)$ is also $A$-invariant and so is completely contained in $\operatorname{MIN}(A)$.

Proof of Lemma 3.4. Let $F$ be a $(n-1)$-flat in $\tilde{M}$ and denote by $U \subset Y$ the image of $F \cap M I N(A)$ under the canonical projection $M I N(A)=E \times Y \rightarrow Y$. $U$ is a convex subset with the property that every geodesic segment $\sigma \subset Y$ which intersects $U$ in more than one point is contained in $U$. If $F$ intersects $\operatorname{MIN}(\gamma)$ and is not $A$-invariant then $F$ intersects some $A$-flat $E \times\{y\}$ transversally. Hence $U \subset Y$ has non-empty interior and therefore $U=Y$. Lemma 1.2 implies that $Y$ is flat. By our assumptions, $Y$ contains two complete non-parallel geodesics. Therefore $Y$ is isometric to the Euclidean plane and $\tilde{M}$ is isometric to Euclidean $n$-space. This implies that $M$ is closed and flat which contradicts the assumption made at the beginning of this section. 
The above result is a key step in our argument, and a very similar result appears in Casson's proof of the Torus Theorem in the 3-dimensional case [C]. In Casson's argument, no assumption is made about the metric on $M$. Instead of considering a totally geodesic immersion of the torus in $M$, he considers a least area immersion. This means that in the universal cover of $M$, he considers area minimizing planes rather than flats. Any two such planes must be disjoint or intersect transversely in a single line. Again this situation is very similar to that in this paper, but double lines of area minimising planes need not be geodesics. Call two of these double lines weakly parallel if there is a non-trivial element of $\pi_{1}(M)$ which stabilises both of them. The analogue of our lemma is his result that either $\pi_{1}(M)$ contains the free abelian group of rank three, so that $M$ is closed and must admit a flat metric, or that all the double lines are weakly parallel.

This result shows that under the hypotheses of 3.4, no $\Gamma$-flat can cross $\partial H_{A}$ transversally, as such a flat would have to be $A$-invariant and so be completely contained in $H_{A}$. With Lemma 3.3, we obtain:

Proposition 3.5. If $\operatorname{MIN}(A)$ contains two non-parallel $A$-invariant $\Gamma$-flats, then $H_{A}$ has non-empty interior and the boundary $\partial H_{A}$ is a disjoint union of isolated A-invariant $\Gamma$-flats.

The quotient of $H_{A}$ by $N(A)$ is a Seifert fibred manifold $S_{A}$ with fibres being closed flat manifolds of dimension $n-2$, and the fibres form a totally geodesic foliation of $S_{A}$. (The definition of Seifert fibered manifolds in arbitrary dimension is given at the end of section 1.) In dimension three, this is a foliation by closed geodesics.

\subsection{The decomposition}

We continue to assume that $M$ is not a closed flat manifold. By Proposition 2.4, there are finitely many families of parallel isolated flat closed hypersurfaces in $M$. In order to avoid unnecessary flat pieces (which are topologically interval bundles over closed flat $(n-1)$-manifolds) in the decomposition of $M$ obtained below, we choose in each family of parallel hypersurfaces a canonical one as follows: For a $\Gamma$-flat $F \subset \tilde{M}$ the set of all $\Gamma$-flats parallel to $F$ splits as a product $F \times I$ where $I$ is a closed connected subset of $\mathbb{R}$. Since $M$ is assumed not to be flat, $I$ is isometric to a compact interval $[-a, a]$.

Definition 3.6. We call the $\Gamma$-flat $F \times\{0\}$ and the immersed hypersurface which it coveres central. We call the isolated $\Gamma$-flat $F \subset \tilde{M}$ and the embedded hypersurface covered by it in $M$ preferred if either $F \subset \partial \tilde{M}$ or $F$ is central and $F \subset \operatorname{Int}(\tilde{M})$.

A preferred $\Gamma$-flat $F$ has the useful property that every $(n-1)$-flat $F^{\prime}$ parallel to $F$ satisfies $\operatorname{Stab}_{\Gamma}\left(F^{\prime}\right) \subseteq \operatorname{Stab}_{\Gamma}(F)$. Accordingly, each isolated closed hypersurface 
can be homotoped to the unique preferred hypersurface parallel to it.

We now consider the finite collection $\mathcal{F}$ of all preferred isolated hypersurfaces in $M$. They are disjoint, embedded and pairwise non-parallel. They decompose $M$ into finitely many pieces which are compact non-flat manifolds with convex boundary. Let $N$ be a piece of the decomposition and denote its fundamental group by $\Gamma^{\prime}:=\pi_{1}(N)$. $N$ has the property that all its preferred isolated flat hypersurfaces are contained in the boundary and hence every isolated closed flat hypersurface can be homotoped into the boundary. We have the following dichotomy:

- All immersed closed flat hypersurfaces are isolated and can be homotoped into the boundary.

- $N$ contains non-isolated closed flat immersed hypersurfaces.

This dichotomy corresponds to the two types of pieces occurring in the topological decomposition theorem in the three-dimensional case, compare section 1. The pieces of the first kind are codimension-one atoroidal. (See section 1 for a definition; in dimension three this is equivalent to being atoroidal.) Assume that $N$ is a piece of the second kind. Then $\tilde{N}$ contains two $\Gamma$-flats $F_{1}$ and $F_{2}$ which intersect transversally in a $(n-2)$-flat $L . \operatorname{Stab}_{\Gamma^{\prime}}\left(F_{1}\right) \cap \operatorname{Stab}_{\Gamma^{\prime}}\left(F_{2}\right)$ acts cocompactly on $L$ by Lemma 2.1, and it contains an abelian subgroup $A$ of finite index and rank $n-2$. According to 3.5, the corresponding Seifert fibered manifold $S_{A}$ has non-empty interior. Each boundary component of $S_{A}$ is an isolated flat hypersurface and can hence be homotoped into $\partial N$. By the construction of $S_{A}$ it follows that $\partial S_{A} \subseteq \partial N$ and therefore $S_{A}=N$. Thus $N$ is a geometric Seifert piece. This concludes the proof of the following result:

Geometric Decomposition Theorem 3.7. Let $M$ be a compact connected nonpositively curved manifold which has convex boundary. Then either $M$ is closed and flat or the following holds.

Let $\mathcal{F}$ be the family of all preferred isolated totally-geodesic closed flat codimension-one submanifolds of $M$. Then $\mathcal{F}$ is a finite collection of disjoint, mutually non-parallel, embedded hypersurfaces and decomposes $M$ into compact manifolds with convex boundary which are Seifert or atoroidal. The Seifert components are foliated by codimension-two totally geodesic closed flat submanifolds and the foliation is locally a Riemannian product foliation. Further any $\pi_{1}$-injective map of a closed flat $(n-1)$-manifold into $M$ can be homotoped to a totally geodesic flat immersion, and any such immersion must lie in one of the Seifert pieces or be parallel to a hypersurface of $\mathcal{F}$. 


\section{Splitting along submanifolds with boundary}

In this section we will state and prove our most general result which corresponds to the full JSJ decomposition of a compact 3-manifold with boundary.

If a Riemannian manifold $\Sigma$ has totally geodesic boundary, we will abbreviate this to say that $\Sigma$ has TGB. A proper map into an $n$-manifold $M$ of a compact flat $(n-1)$-manifold with TGB is essential if it is $\pi_{1}$-injective and not properly homotopic into the boundary of $M$. We will say that $M$ is simple if it does not admit an essential map of a compact flat $(n-1)$-manifold with TGB.

In order to prove our general decomposition theorem, we will consider a compact connected non-positively curved manifold $M$ of dimension $n \geq 3$, which has TGB. This assumption on the boundary means that we can double $M$ along its boundary to obtain a closed connected non-positively curved manifold $D M$ of dimension $n$. If $M$ is not flat, neither is $D M$ and we can apply our main geometric decomposition theorem from the preceding section to obtain the canonical decomposition of $D M$ by finitely many totally-geodesic flat closed submanifolds of codimension one. The fact that this splitting is canonical means that it is invariant under the involution $\tau$ which interchanges the two copies of $M$ in $D M$. Thus the intersection with $M$ of the canonical family of totally geodesic flat closed codimension-one submanifolds of $D M$ yields the required canonical splitting of $M$. The non-simple pieces of $M$ are obtained from the Seifert manifold pieces of $D M$ by intersecting them with $M$. Thus these pieces of $M$ are either Seifert manifolds themselves or they are "half a Seifert manifold". This second case occurs when a Seifert piece $\Sigma$ of $D M$ is $\tau$-invariant, so that the intersection of $\Sigma$ with $M$ consists of half of $\Sigma$. The restriction of $\tau$ to $\Sigma$ is itself an isometry and it fixes $\Sigma \cap \partial M$ pointwise. Thus, for each component $\Omega$ of $\Sigma \cap \partial M$, this isometry of $\Sigma$ lifts to an isometry of the universal cover of $\Sigma$ which fixes pointwise a copy $\Pi$ of the universal cover of $\Omega$. Recall that the universal cover of $\Sigma$ is metrically a product $Z \times E$, where $Z$ is some 2-dimensional space and $E$ is isometric to Euclidean space of dimension $n-2$. Also recall that $\Pi$ is part of a flat in the universal cover of $D M$ and hence is a flat in $Z \times E$. It follows that $\Pi$ is of the form $P \times Q$, where $P$ is some subset of $Z$ and $Q$ is some subset of $E$. Hence $\Sigma \cap \partial M$ is either vertical or horizontal in $\Sigma$, where (as in dimension three) a codimension-one submanifold is vertical if it is a union of fibers of the Seifert structure, and is horizontal if it is transverse to every fibre. In the vertical case, $\Sigma \cap M$ is again a Seifert fiber space. In the horizontal case, $\Sigma \cap M$ must be the product (or twisted product) of $\Sigma \cap \partial M$ with an interval.

General Geometric Decomposition Theorem. Let $M$ be a compact connected non-positively curved manifold of dimension $n \geq 3$, which has TGB. Then either the metric on $M$ is flat, or $M$ can be canonically decomposed along finitely many totally-geodesically properly embedded flat compact submanifolds of codimension one with TGB. The resulting pieces are simple or Seifert fibered or are bundles 
with fibre an interval over a compact $(n-1)$-manifold with TGB. Further any essential map into $M$ of a compact flat $(n-1)$-manifold with TGB can be properly homotoped to a totally geodesic flat immersion, and any such immersion must lie in one of the non-simple pieces or be parallel to one of the canonical surfaces.

\section{References}

[C] A. J. Casson, A new proof of the Torus Theorem, preprint.

[CJ] A. J. Casson, D. Jungreis, Convergence groups amd Seifert fibered 3-manifolds, Invent. Math. 118 (1994), 441-456.

[ChE] J. Cheeger and D. Ebin, Comparison Theorems in Riemannian Geometry, North Holland 1975.

[D] M. J. Dunwoody, Bounding the decomposition of a Poincaré duality group, Bull. London Math. Soc. 21 (1989), 466-468.

[Ga] D. Gabai, Convergence groups are Fuchsian groups, Ann. Math. 136 (1992), 447-510.

[GW] D. Gromoll and J. Wolf, Some relations between the metric structure and the algebraic structure of the fundamental group in manifolds of nonpositive curvature, Bull. AMS $\mathbf{7 7}$, vol. 4 (1971), 545-552.

[G] M. Gromov, Manifolds of negative curvature, J. Diff. Geom. 13 (1978), 223-230.

[H] J. Hempel, 3-Manifolds, Annals of Math. Studies 86, Princeton University Press, Princeton N. J., 1976.

[Ja] W. Jaco, Lectures on three-manifold topology, AMS 1979.

[JS] W. Jaco and P. B. Shalen, Seifert fibred spaces in 3-manifolds, Mem. AMS 220 (1979).

[Jo] K. Johannson, Homotopy equivalences of 3-manifolds with boundary, Springer LNM $\mathbf{7 6 1}$ (1979).

[K] H. Karcher, Riemannian comparison constructions. In: S. S. Chern (ed.), Global Differential Geometry, MAA Studies in Mathematics vol. 27 (1989), 170-222.

[Kr] P. H. Kropholler, An analogue of the torus decomposition theorem for certain Poincaré duality groups, Proc. London Math. Soc. 60 (1990), 503-529.

[LY] H. B. Lawson and S. T. Yau, On compact manifolds of nonpositive curvature, J. Diff. Geom. 7 (1972), 211-238.

[L1] B. Leeb, 3-manifolds with(out) metrics of nonpositive curvature, Ph.D. thesis, Maryland 1992.

[L2] B. Leeb, 3-manifolds with(out) metrics of nonpositive curvature, Invent. Math. 122 (1995), $277-289$.

[RS] E. Rips , Z. Sela, Cyclic splittings of finitely presented groups and the canonical JSJ decomposition, Ann. of Math. 146(2) (1997), 53-109.

[Sc] P. Scott, The geometries of 3-manifolds, Bull. LMS 15 (1983), 401-487.

[S] Z. Sela, Structure and rigidity in (Gromov) hyperbolic groups and discrete groups in rank 1 Lie groups, Geom. Funct. Anal. 7 (1997), 561-593.

Bernhard Leeb

Mathematisches Institut

Universität Tübingen

Auf der Morgenstelle 10

D-72076 Tübingen

Germany

e-mail: leeb@riemann.mathematik.uni-tuebingen.de
Peter Scott

Mathematics Department

University of Michigan

Ann Arbor, MI 48109, USA

e-mail: pscott@umich.edu

(Received: May 20, 1996) 